\title{
Structure of visible and dark matter components in spiral galaxies at redshifts $z=0.5-0.9$
}

\author{
A. Tamm ${ }^{1}$ and P. Tenjes ${ }^{1,2}$ \\ ${ }^{1}$ Institute of Theoretical Physics, Tartu University, Tähe 4, Tartu 51010, Estonia \\ e-mail: [atamm;ptenjes]@ut.ee \\ 2 Tartu Observatory, Torravere, Tartumaa 61602, Estonia
}

Received 23 August 2004 / Accepted 29 November 2004

\begin{abstract}
We have constructed self-consistent light and mass distribution models for four disk galaxies at redshifts $z=0.48$, 0.58, 0.81 and 0.88, using the HST archive WFPC2 observations (HDF-S, MDS, Groth Strip survey) and rotation curves measured by Vogt et al. (1996) and Rigopoulou et al. (2002). The models consist of three components: a bulge, a disk and a dark matter halo. Similarly to the sample studied in Paper I (Tamm \& Tenjes 2003), light distribution of the galaxies in the outer parts is clearly steeper than a simple exponential disk. After applying k-corrections, calculated mass-to-light ratios for galactic disks within the maximum disk assumption are $M / L_{B}=0.9,7.4,4.3$ and 1.4, respectively. Together with the galaxies from Paper I, the mean $\left\langle M / L_{B}\right\rangle=2.5$ at $\langle z\rangle \simeq 0.9$, indicating no significant evolution of $M / L_{B}$ with redshift. Central densities of dark matter halos for an isothermal model are $0.008,0.035,0.013$, and 0.022 in units $M_{\odot} / \mathrm{pc}^{3}$, respectively. Together with the galaxies from Paper I, the DM central density of the four galaxies at mean readshift $\langle z\rangle \simeq 0.9$ is $\rho(0)=(0.012-0.028) M_{\odot} / \mathrm{pc}^{3}$, also showing no significant evolution with redshift.

We have also constructed mass distribution models without assuming flat rotation curves and without a dark matter component. Due to the limited extent of the observed rotation, the models without a dark halo nearly fit the observations. In this case, mass-to-light ratios for the galactic disks are 1.8, 9.7, 12. and 1.9, respectively.
\end{abstract}

Key words. galaxies: photometry - galaxies: fundamental parameters - galaxies: high-redshift - galaxies: spiral galaxies: structure - dark matter

\section{Introduction}

The study of dark matter halo central densities and mass distribution allows us to constrain possible galaxy formation models and large scale structure formation scenarios (Navarro \& Steinmetz 2000; Khairul Alam et al. 2002; Gentile et al. 2004). For this kind of analysis, it is necessary to know both the distribution of visible and dark matter. Unfortunately, the structure and mass distribution of stellar populations, and therefore, the discrimination between visible and dark matter in galaxies (especially in very distant galaxies) is not known precisely enough. Thus, it is interesting to analyze the corresponding data at different redshifts.

In most cases, the evolution of the visible structure of galaxies with redshift has been studied, e.g. the luminosity function (LF), C-M diagram, fundamental plane (FP), the Tully-Fischer (TF) diagram, scale-lengths of galactic disks.

The luminosity function in deep fields has been studied by Gabasch et al. (2004) in the FORS Deep Field up to redshifts $z \sim 5$, Poli et al. (2003) in the HDS-S up to $z \sim 3.5$, and certain evolutionary changes have been found. At the faint end of the LF, no significant evolution has been detected, but at the bright end clear brightening was found. Even at intermediate redshifts $(z<1.5)$ significant evolution was detected at the bright end by Cowie et al. (2004). Toft et al. (2004) studied the LF diagram of an X-ray cluster at $z=1.2$ and in addition to the brightening they found also flattening of the faint end slope of the LF.

The C-M diagram of a large number of galaxies in the redshift range $0.2<z<1.1$ was studied by Bell et al. (2004), and evolutionary trends for both early type and late type galaxies were found. While the early type galaxies evolve only mildly with redshift, the number of luminous late type galaxies increases remarkably with redshift.

The fundamental plane in two clusters $(z=0.58$ and 0.83$)$ was studied by Wuyts et al. (2004). They confirm an earlier result that the slopes of the FP do not change within the studied redshift range. However, there is a general evolutionary shift indicating that galaxies in these clusters formed at $z \approx 3.0$.

To study the evolution of the TF diagram, the maximal rotation velocities in rich cluster galaxies at redshifts $z=0.3-0.5$ were measured by Ziegler et al. (2003). Their analysis did not show any significant difference between cluster and field galaxies at the same redshifts, even if compared to the local galaxies. At higher redshifts $(z=0.2-1.2$, mean $z \simeq 0.7)$, the TF diagram was studied on the basis of 83 disk galaxies by 
Table 1. General galactic parameters.

\begin{tabular}{llllllllll}
\hline \hline Name & RA & $\begin{array}{l}\text { Dec } \\
(2000)\end{array}$ & $\begin{array}{l}\text { Hubble } \\
\text { type }\end{array}$ & $z^{a}$ & $\begin{array}{l}\text { Scale } \\
\left(\mathrm{kpc} /{ }^{\prime \prime}\right)\end{array}$ & $\begin{array}{l}\text { Inclin. }^{a} \\
(\mathrm{deg})\end{array}$ & $\begin{array}{l}m_{I}^{b} \\
(\mathrm{mag})\end{array}$ & $\begin{array}{l}A_{B}^{c} \\
(\mathrm{mag})\end{array}$ & $\begin{array}{l}M_{B}^{c} \\
(\mathrm{mag})\end{array}$ \\
\hline GSS 104-4024 & $14^{\mathrm{h}} 17^{\mathrm{m}} 26.9^{\mathrm{s}}$ & $52^{\circ} 24^{\prime} 49.5^{\prime \prime}$ & $\mathrm{Sbc}$ & 0.812 & 6.52 & 82 & 21.88 & 0.034 & -20.5 \\
GSS 064-4442 & $14^{\mathrm{h}} 17^{\mathrm{m}} 54.0^{\mathrm{s}}$ & $52^{\circ} 29^{\prime} 14.3^{\prime \prime}$ & $\mathrm{Sbc}$ & 0.877 & 6.62 & 55 & 22.05 & 0.033 & -20.4 \\
MDS uem0-043 & $3^{\mathrm{h}} 4^{\mathrm{m}} 59.1^{\mathrm{s}}$ & $-0^{\circ} 11^{\prime} 47.0^{\prime \prime}$ & $\mathrm{Sc}$ & 0.476 & 5.51 & 46 & 19.21 & 0.362 & -21.4 \\
HDFS J223247.66-603335.9 & $22^{\mathrm{h}} 32^{\mathrm{m}} 47.7^{\mathrm{s}}$ & $-60^{\circ} 33^{\prime} 35.9^{\prime \prime}$ & $\mathrm{Sb}(\mathrm{c})$ & 0.581 & 5.95 & 49 & 19.14 & 0.119 & -21.2 \\
\hline
\end{tabular}

${ }^{a}$ Galactic redshifts are from Vogt et al. (1996) and (for HDFS J223247.66-603335.9) Rigopoulou et al. (2002), inclinations are from Simard et al. (2002) and Rigopoulou et al. (2002).

${ }^{b}$ Integrated apparent $I$ magnitudes calculated from our photometry.

${ }^{c}$ Absolute $B$-magnitudes in the galactic rest-frame are calculated from our models (Sect. 4), considering the absorption in the Galaxy $A_{B}$ according to Schlegel et al. (1998).

Table 2. HST observations used for photometry.

\begin{tabular}{lllll}
\hline \hline Name $^{a}$ & $\begin{array}{l}\text { HST survey } \\
\text { program }\end{array}$ & $\begin{array}{l}\text { Total exposure } \\
\text { time in } I(\mathrm{~s})\end{array}$ & $\begin{array}{l}\text { Background } \\
\text { level in } I\end{array}$ & $\begin{array}{l}\text { Background } \\
\text { level in } V\end{array}$ \\
\hline GSS 104-4024 & Groth Strip Survey & 4400 & 5.9 & 6.9 \\
GSS 064-4442 & Groth Strip Survey & 4400 & 6.15 & 6.9 \\
MDS uem0-043 & Medium Deep Survey & 6700 & 13.5 & 23.3 \\
HDFS J223247.66-603335.9 & HDF-South & 113900 & 0.0 & 0.0 \\
\hline
\end{tabular}

Conselice et al. (2004) and also no statistically significant evolution with redshift was detected. On the other hand, MilvangJenses et al. (2003) found that cluster galaxies are brighter by $0.5^{\mathrm{m}}-1^{\mathrm{m}}$ in $B$ color when compared to field galaxies at the same redshift $(z=0.8)$. For field galaxies at redshifts $z=0.1-1.0$ the TF diagram was obtained by Böhm et al. (2004) and brightening of galaxies with redshift depending on galactic mass was discovered.

If, in addition to the surface photometrical data, the rotation velocities of galaxies are also available, galactic structure can be described in greater detail. In several projects, the rotation of distant galaxies has been measured. In addition to the works referred in Paper I, Erb et al. (2003) measured rotation curves (RCs) of 6 very distant $(z>2)$ galaxies. Unfortunately, the extent of these RCs is rather small (less than $1^{\prime \prime}$ ), the maximum rotation values have often not been reached and the models of these galaxies would be uncertain.

In relation to the study of the TF diagram, Ziegler et al. (2003) measured RCs for 13 galaxies in rich clusters at redshifts $z=0.3-0.5$. Of these galaxies $4-5$ objects can be used for mass modeling (the extent of the measured RCs $>2$ ", kinematics more or less regular). The largest sample of RCs at intermediate redshifts was measured by Jäger et al. (2004), where objects in 7 clusters at redshifts $z=0.3-0.6$ were analyzed and several of the derived RCs could be used for mass distribution modeling.

To construct a self-consistent photometrical and dynamical model of a galaxy, both surface photometry and rotation curve data are needed. For galaxies at intermediate redshifts, the typical scale is $4-8 \mathrm{kpc}$ per arcsec and in order to determine the parameters of galactic components, one should use high-resolution Hubble Space Telescope (HST) photometry in addition to the other photometrical data.
In the present study, we continue our modeling of distant galaxies (Tamm \& Tenjes 2003, Paper I). To derive the surface brightness distribution of these galaxies, we used images from the HST archive. RCs were measured by Vogt et al. (1996) and Rigopoulou et al. (2002). General properties of these galaxies are given in Table 1. Galactic names are from the NED database. In the present work we take $H_{0}=$ $65 \mathrm{~km} \mathrm{~s}^{-1} \mathrm{Mpc}^{-1}$ and $q_{0}=0.5$.

\section{Observations and photometry}

We used the HST WFPC2 images of the galaxies for photometry. The images of GSS 104-4024, GSS 064-4442 and MDS uem0-043 were retrieved from the HST archive, HDFS J223247.66-603335.9 is a galaxy on the Hubble Deep Field South, available via the HST web page. General data about the observational programs and the images are presented in Table 2.

We used pipeline-reduced, on-the-flight calibrated images. The Hubble Deep Field South is available with all necessary reduction done, for the other three galaxies we conducted exposure combining, cosmic-ray removal and background removal with IRAF and STSDAS software packages, as described in detail in Tamm \& Tenjes $(2001,2003)$. The resulting images of the galaxies can be seen in Fig. 1.

We used the Lucy-Richardson method to deconvolve point spreading. We calculated point-spread functions of the WFPC2 camera using TinyTim 6.0. The $V$-band image of GSS 104-4024 could not be deconvolved because of too low signal-to-noise and signal-to-background ratios. Isophotes were calculated using the ellipse task of STSDAS. To construct luminosity profiles from the isophotes, we used deconvolved images for the central parts of galaxies and original images for the outer radii. 

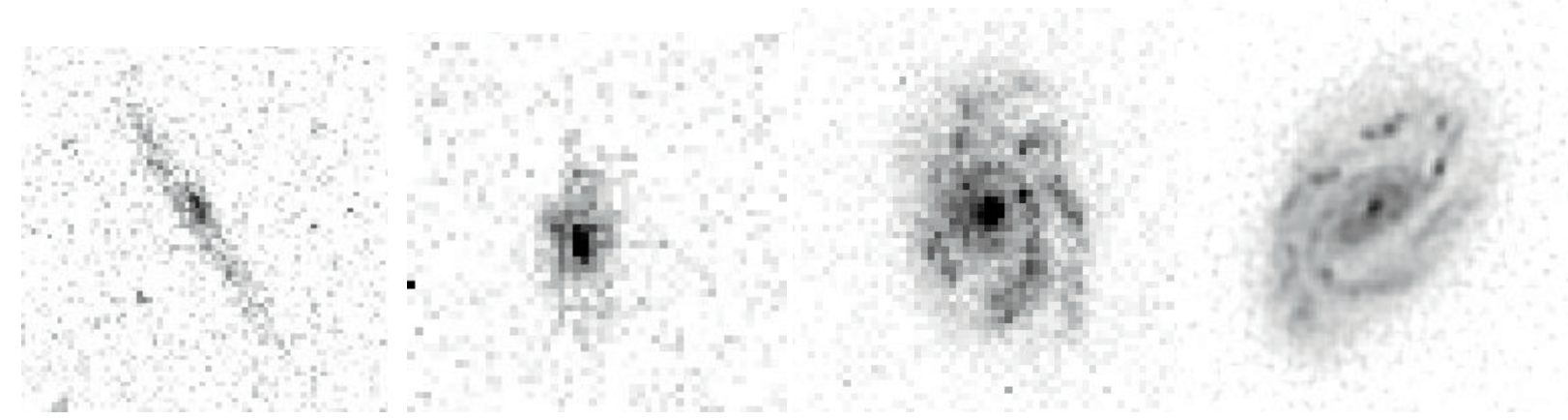

Fig. 1. Images of the galaxies (from left to right) GSS 104-4024, GSS 064-4442, MDS uem0-043 and HDFS J223247.66-603335.9 through $F 814 W$ filter after background subtraction.

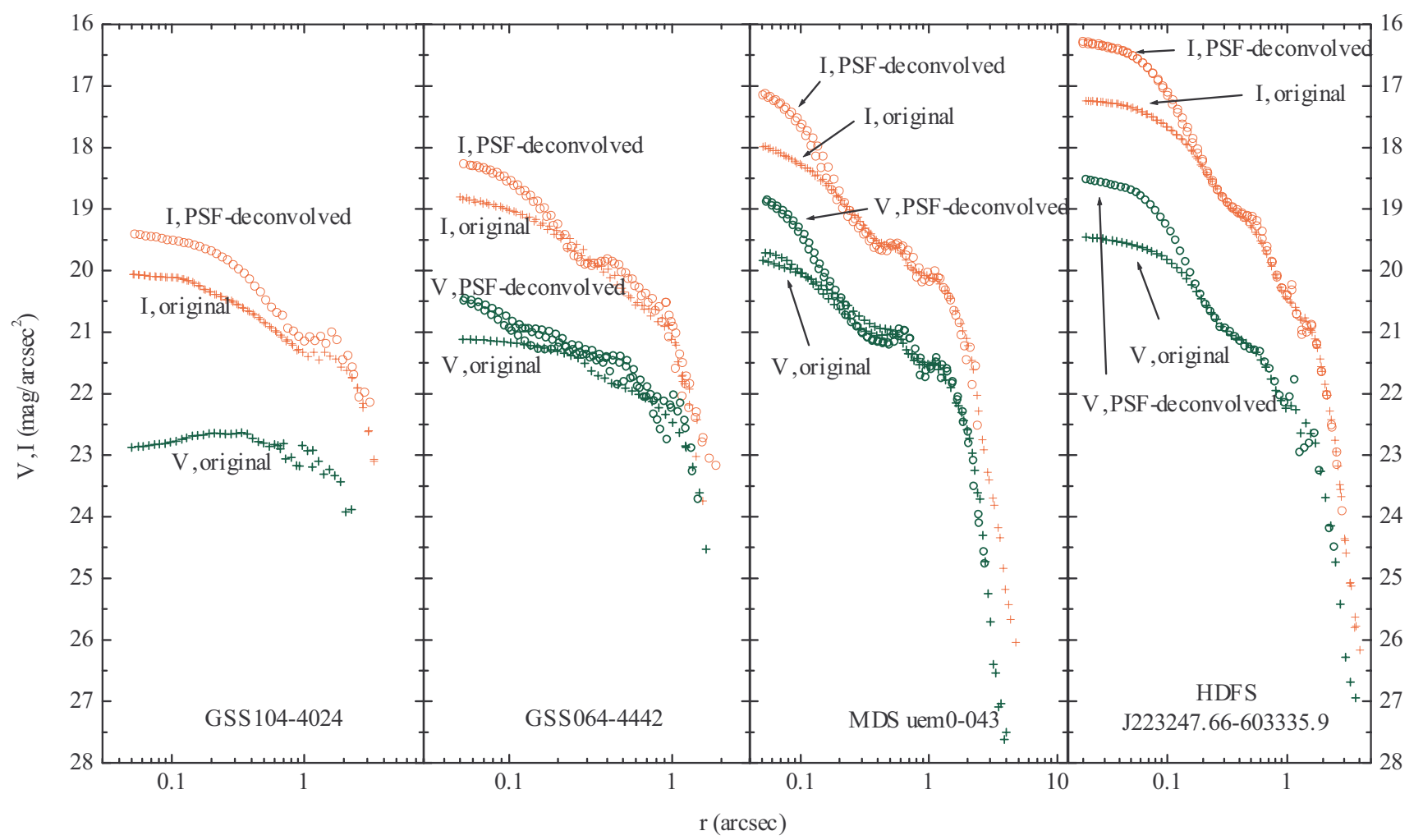

Fig. 2. Surface brightness distribution of the galaxies (from left to right) GSS 104-4024, GSS 064-4442, MDS uem0-043 and HDFS J223247.66-603335.9 in $I$ and $V$ colors in logarithmic distance scale. Both original and deconvolved profiles are given.

We calibrated WFPC2 luminosity counts to Johnson standard magnitudes using formulae and tables given by Holtzman et al. (1995b), see also Tamm \& Tenjes $(2001,2003)$ for details. Luminosity was corrected for the cosmological dimming by a factor of $(1+z)^{4}$. The resulting luminosity profiles of both original and deconvolved images in both $V$ and $I$ passbands are presented in Fig. 2. To present luminosity profiles we prefer a logarithmic scale for distances, enabling a better overview of the deconvolution results. The scatter of the datapoints of different runs of the ellipse task illustrates the uncertainties caused by noise and the limited resolution of the images.

For modeling we derived rest-frame $B$-band luminosities, applying the k-correction according to van Dokkum \& Franx (1996), Kelson et al. (2000). Synthetic spectra were taken from the paper by Coleman et al. (1980), the relation between
Johnson and $A B$-magnitudes from Frei \& Gunn (1994). We determined the following transformations to be conducted

$B(z=0.48)=I+0.585(V-I)+0.7$

$B(z=0.58)=I+0.36(V-I)+0.86$

$B(z=0.81)=I+0.95$

$B(z=0.88)=I-0.106(V-I)+1.18$

The resulting luminosity profiles in the galactic rest-frame $B$ color are shown in Figs. 3 and 4 by open circles. These profiles were used for further modeling.

The RCs for the galaxies GSS 104-4024, GSS 064-4414 and MDS uem0-043, based on Keck spectroscopy, were taken from the paper by Vogt et al. (1996). The velocity profile of HDFS J223247.66-603335.9 was measured by Rigopoulou et al. (2002) using VLT spectra. 


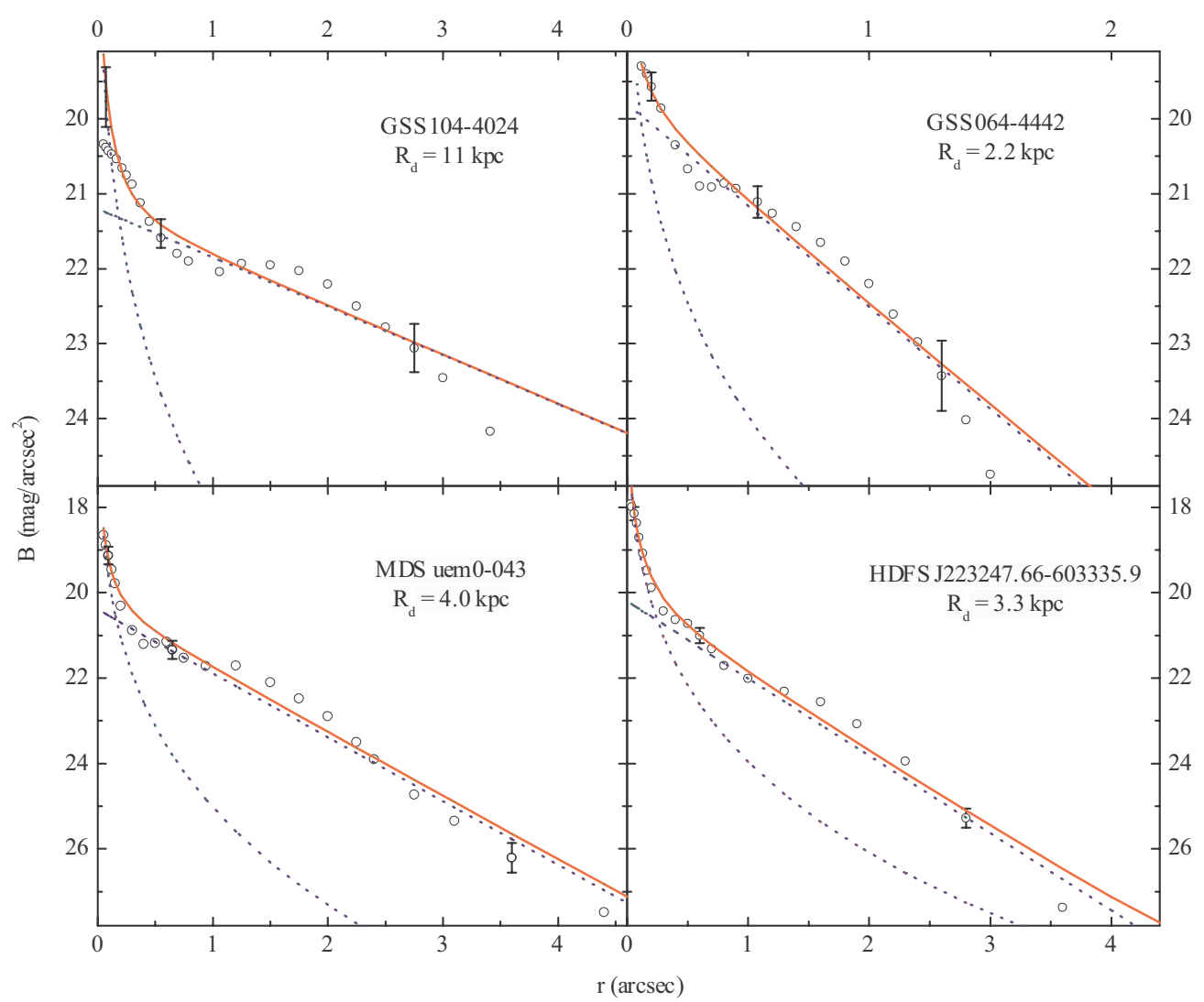

Fig. 3. Surface brightness distribution of the galaxies GSS 104-4024, GSS 064-4442, MDS uem0-043 and HDFS J223247.66-603335.9 in rest-frame $B$ color (open circles). Dashed lines - surface brightness distribution of best fit $r^{1 / 4}$ bulge and exponential disk, solid line - total surface brightness distribution.

We have minimized the difference between the RCs from the two sides of each galaxy, shifting the coordinates $\left(r_{0}, V_{0}\right)$ of the RC panels (see Persic \& Salucci 1995, for details). This makes the RC profiles more regular. The resulting folded RCs are presented with circles in Figs. 5-8. We have used different types of circles (open and closed) to discriminate between the observations in opposite directions from the center. Error bars are also shown. We have tried to select galaxies with kinematics as regular as possible.

\section{Model description}

As in Paper I, we limit the main stellar components to the bulge and the disk. To construct a dynamical model, a dark matter component - the dark halo - must be added to visible components.

To construct a model with two stellar components, the surface luminosity distribution is usually approximated by a $r^{1 / 4}$-bulge and an exponential disk. These surface brightness distributions can be expressed with the help of the general Sérsic formula (1)

$$
I(a)=I(0) \exp \left[-b_{m}\left(a / a_{c}\right)^{1 / m}\right]
$$

(Sérsic 1968) with a parameter $m \geq 2$ for the bulge and $m=1$ for the disk. In this formula $a$ is distance along the galactic major axis, $a_{c}$ is the radius containing half of the total luminosity, $b_{m}$ is a normalizing constant.
If, in addition to the photometrical data, kinematic data are also used, the corresponding dynamical model must be consistent with the photometry, i.e. the same density distribution law must be used for RC modeling (and for the velocity dispersion curve, if possible). In the case of an infinitely thin disk, the circular velocity for an exponential surface density distribution can be expressed via modified Bessel functions (Freeman 1970). In addition, for spherical systems an expression for circular velocity with an integer Sérsic index $m$ (Mazure \& Capelato 2002) can be derived. For a non-integer index and ellipsoidal surface density distribution a consistent solution for $\mathrm{RC}$ calculations is not known.

The density distribution parameters are often determined by the least squares method. In this case the parameter $m$ is not necessarily an integer number. In the present paper, the density distribution parameters are determined by the least squares method and they can have any value. Moreover, we have found that at least for our present sample of high redshift galaxies the best fit for the disk luminosity distribution is achieved with the Sérsic index $m<1$.

For the reasons given above we decided in addition to models with a $r^{1 / 4}$ bulge and exponential disk to construct models for the galaxies starting from a spatial density distribution law, which allows an easier least-square fitting simultaneously for light distribution and RC.

In such models, the visible part of a galaxy is given as a superposition of a bulge and a disk. The spatial density distribution of each visible component is approximated by an 


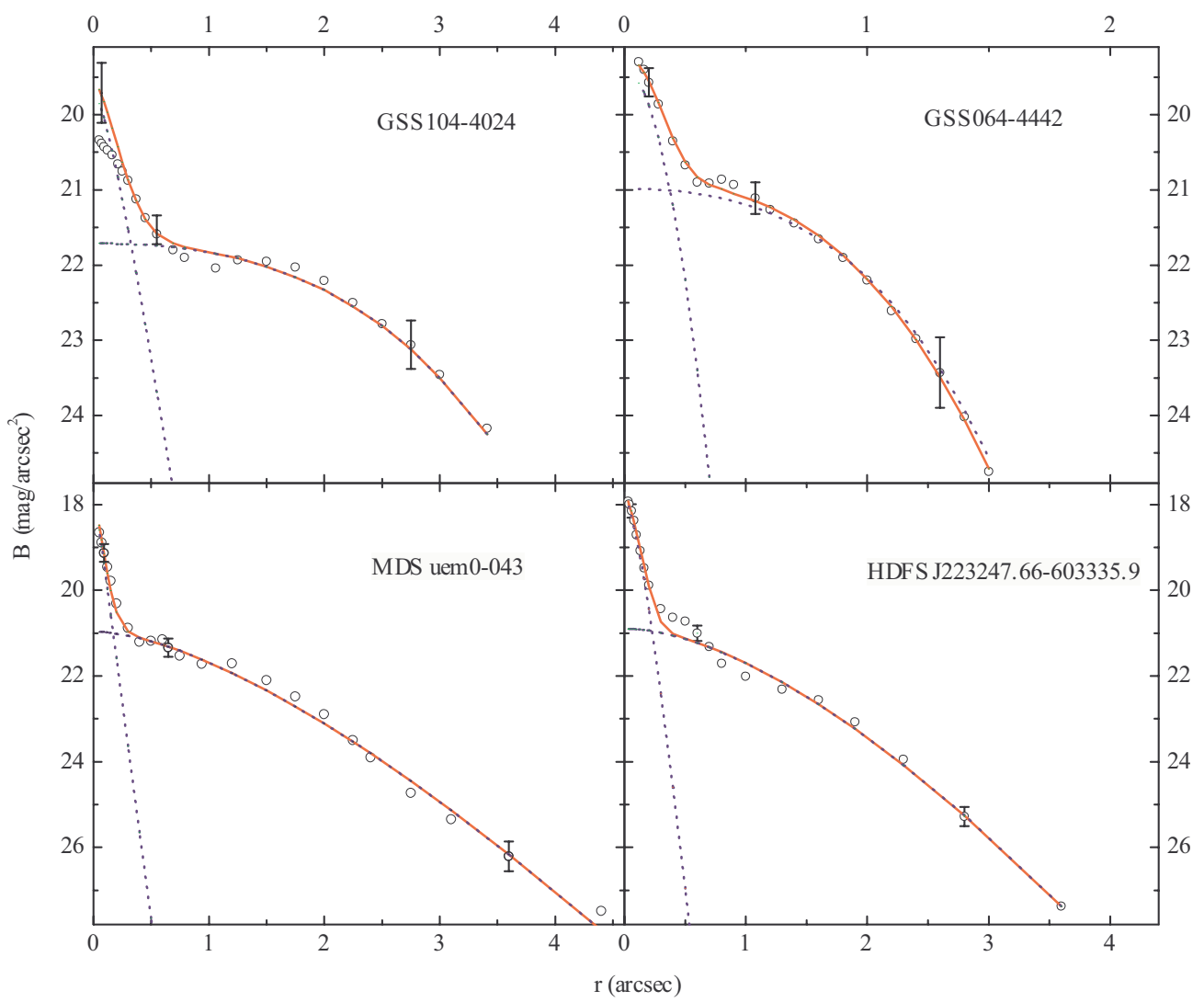

Fig. 4. Surface brightness distribution of the galaxies GSS 104-4024, GSS 064-4442, MDS uem0-043 and HDFS J223247.66-603335.9 in rest-frame $B$ color (open circles). Dashed lines - surface brightness distribution of best fit model components using spatial density distribution (2). Solid line - total surface brightness.

inhomogeneous ellipsoid of rotational symmetry with the constant axial ratio $q$ and the density distribution law

$\rho(a)=\rho(0) \exp \left[-\left(a /\left(k a_{0}\right)\right)^{1 / N}\right]$

where $\rho(0)=h M /\left(4 \pi q a_{0}^{3}\right)$ is the central density and $M$ is the component mass; $a=\sqrt{R^{2}+z^{2} / q^{2}}$, where $R$ and $z$ are two cylindrical coordinates. $a_{0}$ is the harmonic mean radius which characterizes rather well the real extent of a component, independently of the parameter $N$. Coefficients $h$ and $k$ are normalizing parameters, depending on $N$, which allows the density behavior to vary with $a$. The definition of the normalizing parameters $h$ and $k$ and their calculation is described in Tenjes et al. (1994). Equation (1) allows a sufficiently precise numerical integration and has a minimum number of free parameters.

The dark matter (DM) distribution is represented by a spherical isothermal law

$\rho(a)= \begin{cases}\rho(0)\left(\left[1+\left(\frac{a}{a_{c}}\right)^{2}\right]^{-1}-\left[1+\left(\frac{a^{0}}{a_{c}}\right)^{2}\right]^{-1}\right) & a \leq a^{0} \\ 0 & a>a^{0} .\end{cases}$

Here $a^{0}$ is the outer cutoff radius of the isothermal sphere, $a_{c}=$ $k a_{0}$.

The density distributions for the bulge and the disk were projected along the line of sight, divided by their mass-to-luminosity ratios $f$ and their sum gives us the surface brightness distribution of the model

$L(A)=2 \sum_{i=1}^{2} \frac{q_{i}}{Q_{i} f_{i}} \int_{A}^{\infty} \frac{\rho_{i}(a) a \mathrm{~d} a}{\left(a^{2}-A^{2}\right)^{1 / 2}}$,

where $A$ is the major semiaxis of the equidensity ellipse of the projected light distribution and $Q_{i}$ are their apparent axial ratios $Q^{2}=\cos ^{2} \gamma+q^{2} \sin ^{2} \gamma$. The angle between the plane of a galaxy and the plane of the sky is denoted by $\gamma$. The summation index $i$ designates two visible components, the bulge and the disk.

The masses of the components were determined from the rotation law

$v_{i}^{2}(R)=4 \pi q_{i} G \int_{0}^{R} \frac{\rho_{i}(a) a^{2} \mathrm{~d} a}{\left(R^{2}-e_{i}^{2} a^{2}\right)^{1 / 2}}$,

$V^{2}(R)=\sum_{i=1}^{3} v_{i}^{2}(R)$,

where $G$ is the gravitational constant, $e=\sqrt{1-q^{2}}$ is eccentricity, and $R$ is the distance in the equatorial plane of the galaxy. Now the summation is over all three components.

We have kept the axial ratio of the components fixed, taking $q=0.7$ for the bulge and $q=0.1$ for the disk, according to analogy with the near-by galaxies. The model parameters $a_{0}, L_{B}, M$ and $N$ for the bulge and the disk were determined by a subsequent least-squares approximation process. First, we made a 


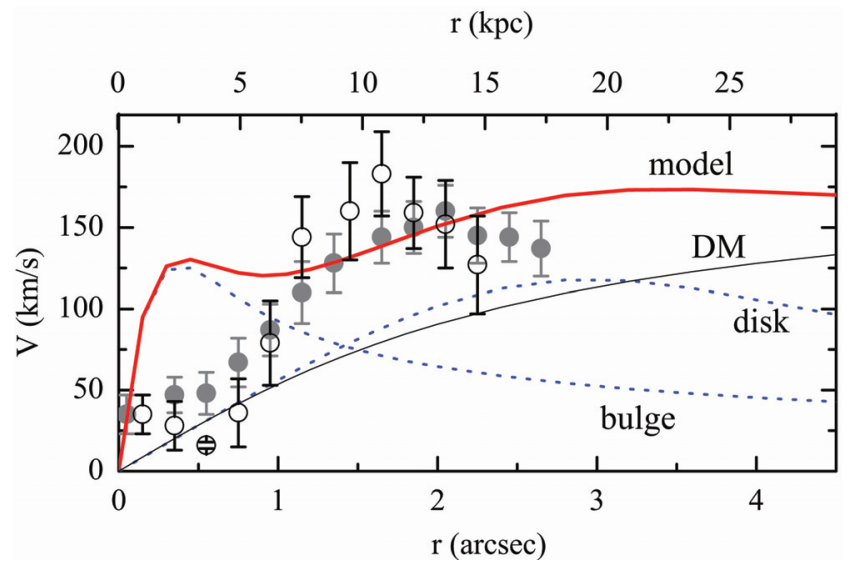

Fig. 5. Rotation curve of the galaxy GSS 104-4024. Open and gray circles - observed velocities by Vogt et al. (1996) at the opposite sides of the galaxy, thick solid line - rotation curve from the mass model, dashed lines - rotation velocities due to visible components, thin solid line - rotation velocities due to the dark matter component.

crude estimation of the population parameters. The purpose of this step is to avoid obviously non-physical parameters - relations (4) and (5) are nonlinear and fitting of the model to the observations is not a straightforward procedure. Next, a mathematically correct solution was found for each galaxy. Details of the least squares approximation and the general modeling procedure were described by Einasto \& Haud (1989), Tenjes et al. (1994, 1998).

The RCs of the modeled galaxies have a rather small extent (see Figs. 5-8). We do not have any additional information about the dynamics of these galaxies at large galactocentric radii. For this reason, the outer cutoff radius of the DM component remains undetermined at present. As in Paper I, we fixed $a^{0}=5 a_{0}$ on the basis of the structure of nearby galaxies. This value influences the behavior of the RC only in the extreme outer regions and does not influence our results. In addition, by analogy with nearby galaxies we assumed that the RCs of these galaxies remain nearly flat at least up to $30 \mathrm{kpc} \simeq 1.5-2 R_{\mathrm{opt}}$ (Persic et al. 1996; Sofue \& Rubin 2001).

As we have no information about velocity dispersions in the central regions of our galaxies, we can calculate only the circular velocities (Eqs. (5)-(6)). The difference between the circular and rotational velocities is known in galactic dynamics as the asymmetric drift and is mainly a function of velocity dispersions. Typical emission-line dispersions in disk galaxies at intermediate redshifts are $30-100 \mathrm{~km} \mathrm{~s}^{-1}$ or even more (Im et al. 2001; Erb et al. 2003). Thus, within the central 0.5" $-0.7^{\prime \prime}$, modeled velocities must be higher than the observed rotational velocities (see also Sect. 4).

\section{Results}

We have fitted the two-component models of the galaxies to the $B$ color profiles. Profiles fitted by a $r^{1 / 4}$-bulge and an exponential disk can be seen in Fig. 3. Profiles fitted on the basis of the spatial density law (2) are presented in Fig. 4. In both cases dotted lines correspond to the individual components - the bulge and the disk. The solid line is the sum of the two components.

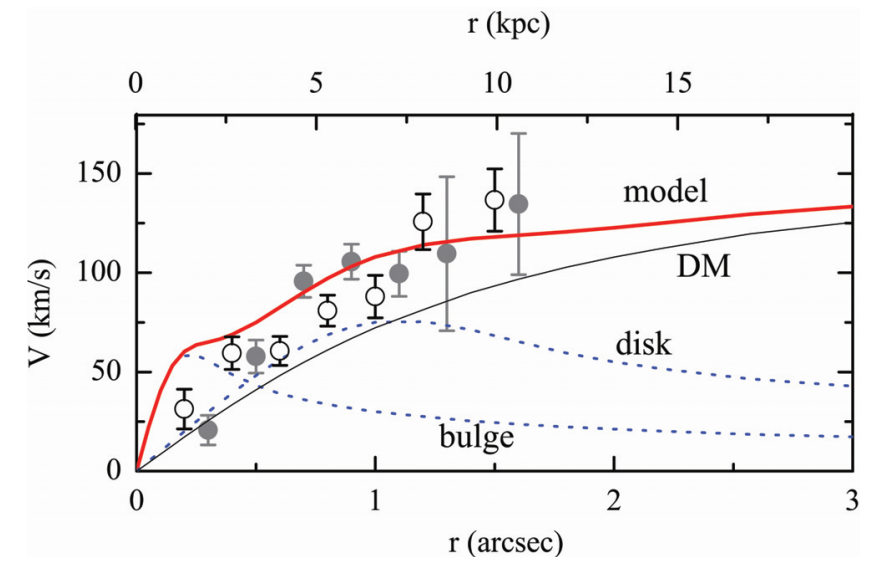

Fig. 6. Rotation curve of the galaxy GSS 064-4442. Designations are the same as in Fig. 5.

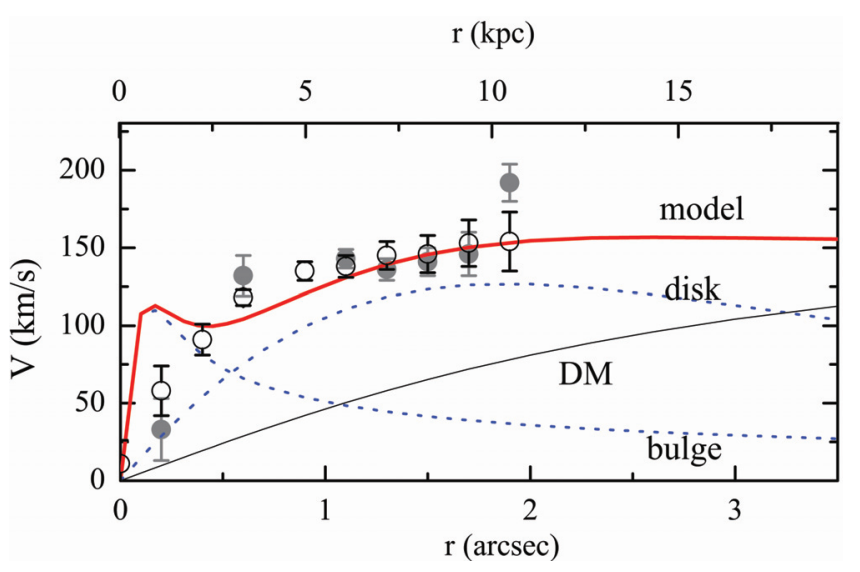

Fig. 7. Rotation curve of the galaxy MDS uem0-043. Designations are the same as in Fig. 5.

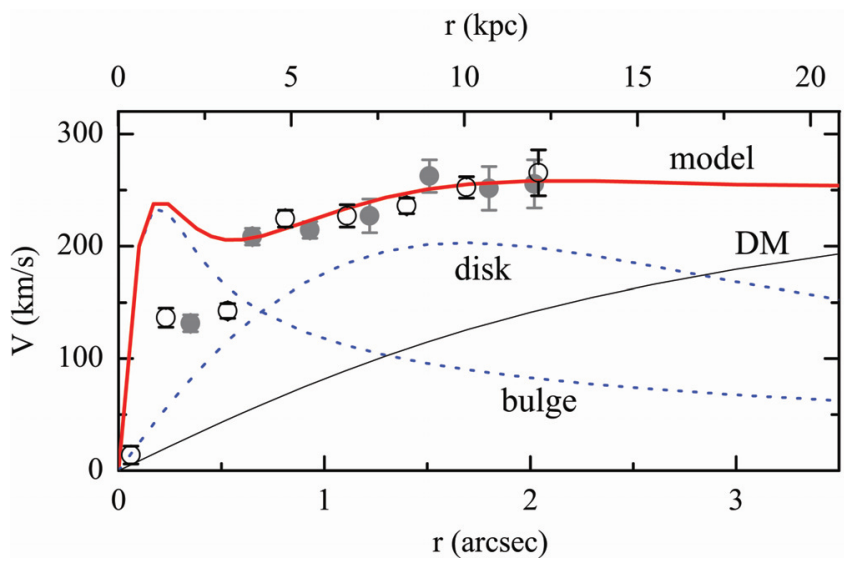

Fig. 8. Rotation curve of the galaxy HDFS J223247.66-603335.9. Designations are the same as in Fig. 5.

It is seen that a non-exponential disk gives a better agreement with observations. The mean deviations of the models from the observations in Fig. 4 are only $\left\langle\mu^{\text {obs }}-\mu^{\text {model }}\right\rangle=0.07^{\mathrm{m}}, 0.04^{\mathrm{m}}$, $0.14^{\mathrm{m}}$ and $0.13^{\mathrm{m}}$, respectively.

Fitting of the modeled RCs to the observations is much more complex. First, the resolution of the present-day spectroscopic observations is insufficient to construct high-precision 
velocity profiles for galaxies at such high redshifts. In addition to the uncertainties and the low extent of the observations, the velocities in the central regions remain severely underestimated due to the lack of resolution and therefore the bulge mass remains practically unknown.

It is possible to fit only the share of the disk or the dark matter component to the observed rotation and still achieve satisfactory concordance. In order to keep the results more realistic we have, however, constructed three-component models for all four galaxies with a bulge, a disk and a dark matter component. For the reasons given above, the mass of the bulge component should not be taken too literally; we have practically ignored the observed velocities at low radii. As in most cases no other hint of the mass of the bulge was given, we took the mass-to-light ratio of the bulge equal to that of the disk. Assuming the bulge of a galaxy to be populated by stars from an older generation than the disk, we should thereby get the approximate lower limit of the bulge mass.

We have scaled the original observed RCs to match $V_{\max }$ given by the authors, thereby compensating for the effects of misalignment of the slit, "beam smearing" and seeing at the outer radii, which we have used to fit our modeled RC.

The parameters of the DM component cannot be determined uniquely by our method. We could, for example, increase its mass, if we increased $r$ simultaneously. A parameter that can be determined reasonably well, however, is the central density of the dark matter $\rho(0)$ (see Sect. 5).

\subsection{Notes about individual galaxies}

\subsubsection{GSS 104-4024}

This Sbc galaxy at $z=0.8116$ is seen nearly edge-on (inclination $82^{\circ}$ ); a possible dust lane along its semi-major axes can be detected on the HST image (Fig. 1), obscuring the central part of the bulge. The usual isophote fitting would underestimate the luminosity of the central regions $\left(r<0.3^{\prime \prime}\right)$; the true luminosity remains unknown. A possible correction suggested by our modeling software is shown in Fig. 4 (the correction contributes only $0.1^{\mathrm{m}}$ to the total luminosity).

We calculated the total apparent $V$-band luminosity of the galaxy to be $m_{I}=21.88 \pm 0.06$, which matches well with the estimate 22.05 by Simard et al. (2002). The mean apparent color index $(V-I)=2.1 \pm 0.4$. Taking into account the absorption in the Galaxy, we found the absolute $B$-band luminosity to be $M_{B}=-20.5$. However, considering also the internal absorption, the galactic luminosity would be higher and the resulting $M / L$ ratio of visible matter would be smaller. For the exponential bulge we find $r_{\mathrm{d}}=11 \mathrm{kpc}$, which coincides with the value 11.54 given by Simard et al. (2002).

\subsubsection{GSS 064-4442}

This is an Sbc galaxy at $z=0.877$, the most distant one discussed in this paper. We calculated the total apparent Johnson $m_{I}=22.05 \pm 0.14$, which matches well with the estimated value 21.97 by Simard et al. (2002). The mean apparent color index $(V-I)=1.5 \pm 0.2$, absolute $B$-band magnitude
$M_{B}=-20.4$. We obtained $r_{\mathrm{d}}=2.2 \mathrm{kpc}$ for the exponential disk, which somewhat differs from the estimate 3.76 by Simard et al. (2002). Taking into account the faintness and low resolution of the image of the galaxy this deviation is not surprising.

\subsubsection{MDS uem0-043}

This well-exposed Sc galaxy at $z=0.476$ has an obvious spiral structure. We found the total Johnson I-band luminosity to be $19.21 \pm 0.04$, which agrees well with the result of Vogt et al. (1996) $m_{I}=19.31$. The mean apparent color in$\operatorname{dex}(V-I)=1.5 \pm 0.2$. With correction for the absorption in the Galaxy applied, MDS uem0-043 turns to be a bright galaxy: $M_{B}=-21.4$. We estimated $r_{\mathrm{d}}=4.0 \mathrm{kpc}$ for the exponential disk, which is reasonably close to $r_{\mathrm{d}}=5.24$ by Vogt et al. (1996). However, in Vogt et al. (1996) deconvolution of the surface brightness profile has not been done, thus a slight difference in disk scale is normal.

\subsubsection{HDFS J223247.66-603335.9}

This bright and massive Sb galaxy is very well exposed on the HDF-South image and was studied in a paper by Rigopoulou et al. (2002). The excellent quality of the HDF images makes it possible to analyze the photometry of the galaxy with high precision. We calculated the total apparent Johnson $I$ luminosity to be $19.14 \pm 0.02$. Transforming this result to the $A B$ system (adding $0.35 \mathrm{mag}$, Frei \& Gunn 1994), we get a perfect match with the estimate of 19.5 by da Costa et al. (1998) on the basis of ground-based observations with the VLT. The mean apparent color index $(V-I)=2.0 \pm 0.2$. The absolute $B$-band luminosity is $M_{B}=-21.2$. We obtained $r_{\mathrm{d}}=3.3 \mathrm{kpc}$ for the exponential disk, which unfortunately cannot be compared to the value by Rigopoulou et al. (2002) due to different surface brightness scaling.

The velocity profiles are presented in Fig. 8. Calculated from our model, the total mass within $28 \mathrm{kpc}$ is $13 \times 10^{11} M_{\odot}$, which agrees well with the mass $10 \times 10^{11} M_{\odot}$ within $20 \mathrm{kpc}$ estimated by Rigopoulou et al. (2002).

\subsection{Calculated model parameters}

In Table 3, calculated from the models, the integrated parameters of a Sérsic surface density distribution are given: the scale lenghts of the bulge $r_{\mathrm{b}}$ and the disk $r_{\mathrm{d}}$ and the corresponding central luminocities in rest-frame $B$-band $\left(\mu_{B}^{0}\right)_{\text {bulge }},\left(\mu_{B}^{0}\right)_{\text {disk }}$. The corresponding profiles can be seen in Fig. 3 .

The parameters of the final models on the basis of the spatial density distribution (2) (the harmonic mean radius $a_{0}$, the total mass of the population $M$, the structural parameter $N$, the dimensionless normalizing constants $h$ and $k$, $B$-luminosities and the corresponding mass-to-light ratios) are given in Table 4. The masses of the bulge and DM are given in parentheses to indicate that on the basis of the present observational data it is not possible to determine these values uniquely. The final model profiles are denoted by thick solid lines in Figs. 4-8. Models of individual visible components are 
Table 3. Derived parameters for a Sérsic surface density distribution.

\begin{tabular}{lllll}
\hline \hline Name $^{a}$ & $\begin{array}{l}r_{\mathrm{b}} \\
(\mathrm{kpc})\end{array}$ & $\begin{array}{l}r_{\mathrm{d}} \\
(\mathrm{kpc})\end{array}$ & $\begin{array}{l}\left(\mu_{B}^{0}\right)_{\text {bulge }} \\
(\mathrm{mag})\end{array}$ & $\begin{array}{l}\left(\mu_{B}^{0}\right)_{\text {disk }} \\
(\mathrm{mag})\end{array}$ \\
\hline GSS 104-4024 & 2.0 & 11.0 & 14.0 & 21.2 \\
GSS 064-4442 & 0.3 & 2.2 & 14.5 & 19.8 \\
MDS uem0-043 & 0.23 & 4.0 & 13.0 & 20.4 \\
HDFS J223247.66-603335.9 & 0.3 & 3.3 & 12.7 & 20.2 \\
\hline
\end{tabular}

presented by dashed lines, the model of the dark matter component by a thin solid line.

In Table 5 the total mass of the visible matter $M_{\text {vis }}$, the total intrinsic luminosity $L_{B}$, the mass-to-light ratio of the visible matter $M / L_{B}$ and the disk-to-bulge ratio $D / B$ are given.

\section{Discussion}

We have used the isothermal approximation for the dark matter density distribution in our models. However, several other dark matter distributions have been suggested and used by different authors. The main debate has been between "cuspy" distributions deduced from $N$-body simulations, with the density increasing infinitely towards the center of the halo, and "cored" distributions usually derived from the observed RCs of late type and low surface brightness galaxies with a constant density distribution in the central part of the halo.

According to their numerical simulations, Navarro et al. (1996, NFW) proposed the following formula for the density distribution

$\rho(r)=\frac{\rho_{\mathrm{s}}}{\left(r / r_{\mathrm{s}}\right)\left[1+\left(r / r_{\mathrm{s}}\right)\right]^{2}}$,

where $\rho_{\mathrm{s}}$ and $r_{\mathrm{s}}$ are the characteristic density and radius, respectively. Moore et al. (1999) proposed a rather similar, but still "cuspier" distribution:

$\rho(r)=\frac{\rho_{\mathrm{s} M}}{\left(r / r_{\mathrm{s}}\right)^{1.5}\left[1+\left(r / r_{\mathrm{s}}\right)^{1.5}\right]}$.

Burkert (1995) studied low surface-brightness galaxies and approximated the dark component with a distribution similar to isothermal

$\rho(r)=\frac{\rho_{0} r_{0}^{3}}{\left(r+r_{0}\right)\left(r^{2}+r_{0}^{2}\right)}$.

We have constructed a "best estimate" curve for the velocity distribution of the galaxy MDS uem0-043, using the observed rotation for the inner radii and keeping the rotation velocities beyond them constant. Thereafter we subtract the share of the modeled visual components from the resulting curve, revealing an estimate for the share of the DM component $V_{\mathrm{DM}}^{2}=V_{\mathrm{obs}}^{2}-V_{\mathrm{vis}}^{2}$. These velocities are shown as open circles in Fig. 9 together with the estimated errors and they correspond to the supposed rotation contribution of DM. Finally, we test how well different density distributions fit with the derived DM contribution. The large uncertainties at small radii are caused by the effects of limited spectral and spatial resolution.

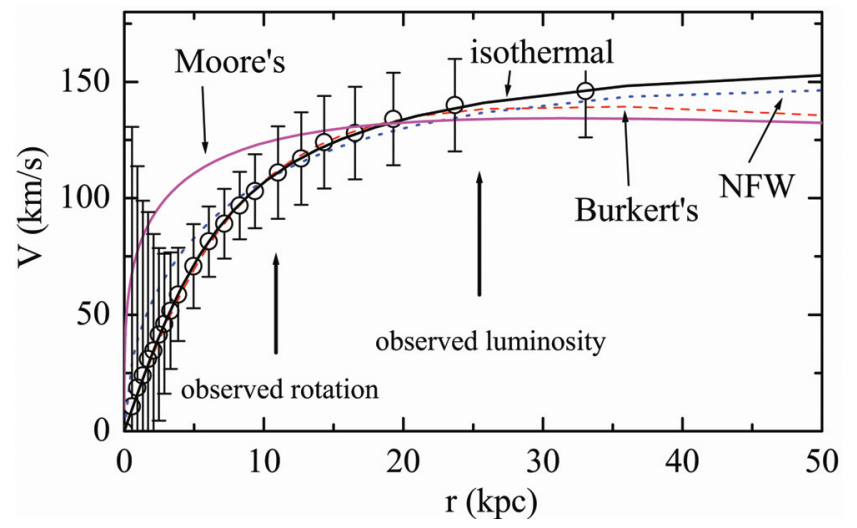

Fig. 9. Comparison of the dark matter contribution in kinematics of the galaxy MDS uem0-043 (open circles with estimated error bars) with the different dark matter density distribution models. Two vertical arrows indicate outermost observed points of the surface brightness profile and the rotation curve.

Except for the "cuspiest" one by Moore et al. (1999), the curves of all other distributions fit within the error bars (Fig. 9). Burkert's model is nearly the same as our isothermal law in the central part, while the best fit of NFW stays slightly above these two at small radii and below them at higher radii. Due to the limited spatial resolution of the RCs of high redshift galaxies, it is not possible to prefer the NFW, Burkert or isothermal distributions, nor to study the most recent profiles by Navarro et al. (2004) and Fukushige et al. (2004). We intend do this analysis on the basis of detailed models of nearby well-studied galaxies.

As the behavior of the density distributions (7) and (8) at the center is limited by the resolution of $N$-body calculations and the behavior of density distributions (3) and (9) is quite similar (Fig. 9), below we discuss only the isothermal DM halo.

Due to the limited extent of RCs it is not possible to determine separately the radius and the mass of the DM distribution. However, the combination of these two parameters, the central density of the dark matter $\rho(0)=h M /\left(4 \pi a_{0}^{3}\right)$, characterizing the central rotation velocity gradient caused by the dark halo, is nearly independent of the choice of the dark halo outer cutoff radius and can be determined. Calculated from our models, the central densities of DM components are $\rho(0)=0.013,0.022$, 0.008 and 0.035 in units $M_{\odot} / \mathrm{pc}^{3}$. These values are calculated for maximum disk models and are thus the lower limits. Adding the two galaxies at redshifts 0.9 and 0.99 from Paper I we get the range of the DM central density of the four galaxies at the mean readshift $\langle z\rangle \simeq 0.9$ is $\rho(0)=(0.012-0.028) M_{\odot} / \mathrm{pc}^{3}$. This value can be compared with the mean central DM density of nine local galaxies $\rho(0)=(1-4) \times 10^{-24} \mathrm{~g} / \mathrm{cm}^{3}=$ (0.015-0.050) $M_{\odot} / \mathrm{pc}^{3}$ derived by Borriello \& Salucci (2001). Although our sample of galaxies is small, there seems to be no significant evolution of DM central density with redshift.

We also calculated the amount of DM within a half-light radius. For galaxies at redshifts $\langle z\rangle \simeq 0.9$ the mean dark matter contribution was $40 \pm 15$ per cent. Thus in our case, the discrepancy between the observed galaxies and CDM models 
Table 4. Model parameters for spatial density distribution.

\begin{tabular}{|c|c|c|c|c|c|c|c|}
\hline Name & $\begin{array}{l}a_{0} \\
(\mathrm{kpc})\end{array}$ & $\begin{array}{l}M \\
\left(10^{10} M_{\odot}\right)\end{array}$ & $\begin{array}{l}M / L_{B} \\
\left(M_{\odot} / L_{\odot}\right)\end{array}$ & $\begin{array}{l}L_{B} \\
\left(10^{10} L_{\odot}\right)\end{array}$ & $N$ & $h$ & $k$ \\
\hline \multicolumn{8}{|c|}{ GSS 104-4024 } \\
\hline Bulge & 1.5 & $(2.2)$ & 4.3 & 0.50 & 0.91 & 3.33 & 0.594 \\
\hline Disk & 13.2 & 8.7 & 4.3 & 2.03 & 0.32 & 1.18 & 1.37 \\
\hline Dark matter & 80. & $(550)$. & & & & 14.8 & 0.151 \\
\hline \multicolumn{8}{|c|}{ GSS 064-4442 } \\
\hline Bulge & 0.9 & $(0.3)$ & 0.90 & 0.31 & 0.51 & 1.59 & 1.12 \\
\hline Disk & 5.0 & 1.8 & 0.90 & 2.03 & 0.34 & 1.23 & 1.34 \\
\hline Dark matter & 50. & (230) & & & & 14.8 & 0.151 \\
\hline \multicolumn{8}{|c|}{ MDS uem0-043 } \\
\hline Bulge & 0.5 & $(0.6)$ & 1.41 & 0.41 & 1.11 & 4.96 & 0.405 \\
\hline Disk & 7.2 & 7.1 & 1.41 & 5.02 & 0.73 & 2.38 & 0.805 \\
\hline Dark matter & 80. & (340.) & & & & 14.8 & 0.151 \\
\hline \multicolumn{8}{|c|}{ HDFS J223247.66-603335.9 } \\
\hline Bulge & 0.7 & (5.6) & 7.4 & 0.75 & 0.80 & 2.70 & 0.719 \\
\hline Disk & 7.0 & 28.3 & 7.4 & 3.83 & 0.67 & 2.12 & 0.888 \\
\hline Dark matter & 80. & $(1500)$. & & & & 14.8 & 0.151 \\
\hline
\end{tabular}

Table 5. Derived integrated parameters from models.

\begin{tabular}{lllll}
\hline \hline Name & $M_{\text {vis }}$ & $L_{B}$ & $M / L_{B}$ & $D / B$ \\
& $10^{10} M_{\odot}$ & $10^{10} L_{\odot}$ & & \\
\hline GSS 104-4024 & 11. & 2.53 & 4.3 & 4.0 \\
GSS 064-4442 & 2.1 & 2.34 & 0.9 & 6.5 \\
MDS uem0-043 & 7.6 & 5.43 & 1.4 & 12.2 \\
HDFS J223247.66-603335.9 & 34. & 4.58 & 7.4 & 5.1 \\
\hline
\end{tabular}

is not as high as was indicated in the analysis by Bell et al. (2003).

According to our models, the mass-to-light ratios of visible matter (within the maximum disk assumption) are $M / L_{B}=$ 4.3, 0.9, 1.4 and 7.4. Adding the two galaxies at redshifts 0.9 and 0.99 from Paper I to these galaxies, we find that the mean $M / L_{B}$ of the four galaxies at the mean redshift $\langle z\rangle \simeq 0.9$ is $\left\langle M / L_{B}\right\rangle=2.5$. Another way to determine stellar masses and mass-to-light ratios is from broadband optical-NIR photometry and chemical evolution models (Drory et al. 2004; Berta et al. 2004). For galaxies at redshifts $z \sim 0.9$ Drory et al. (2004) derived $\left\langle M / L_{B}\right\rangle=1.3$, being somewhat smaller than the value calculated by us. For the highest mass galaxies at redshift $z \sim 0.5$, they derived $\left\langle M / L_{B}\right\rangle=2.6$, also smaller than our value of 7.4 for the galaxy HDFS J223247.66-603335.9. However, we think that within the precision of the models used these results agree reasonably. Stellar masses of starburst galaxies at $z=0.5$ are found to be even as large as $50 \times 10^{10} M_{\odot}$ (Drory et al. 2004; Berta et al. 2004).
The derived $M / L_{B}=2.5$ at $z \sim 0.9$ can be compared to the mean stellar $M / L_{B}$ calculated by Giraud (1998) for local $\mathrm{Sb}$ galaxies $M / L_{B}=2.7$. No significant evolution with redshift is detected - a result which was found earlier in the studies of the TF diagram at intermediate redshifts (Vogt et al. 1996; Ziegler et al. 2003; Conselice et al. 2004; Böhm et al. 2004, for higher mass galaxies). An explanation for these small changes can be found in chemical evolution models. Evolution calculations by Bicker et al. (2004, Fig. 7) indicate that the $\mathrm{k}$-correction and evolution corrections in the $B$-band nearly compensate each other in the case of $\mathrm{Sb}$ galaxies at redshifts $z \simeq 0.5-1$.

As mentioned in Paper I, the disk concentration parameter $N$ seems to have a trend towards lower values at higher redshifts. Even visual inspection of the luminosity profiles (Figs. 2-4) reveals that the surface brightness of the outer parts of the galaxies decreases faster than exponentially. Surface brightness distribution of disks with the concentration parameter $N<1$ is similar to the surface brightness distribution of truncated disks described by Pohlen et al. (2000, 2004). According to recent SPH simulations, truncated disks seem to be a natural phenomena within $\Lambda \mathrm{CDM}$ models (Governato et al. 2004, Fig. 2). However, in our case the truncation takes place generally slightly earlier than at 3-5 disk scale lengths. Truncation of a sample of redshift $z \sim 1$ disk galaxies has recently been studied by Pérez (2004).

It is possible to construct mass distribution models for galaxies without assuming the existence of a DM component. The corresponding models are given in Fig. 10. Now the calculated RCs start to decrease significantly near or right after the 


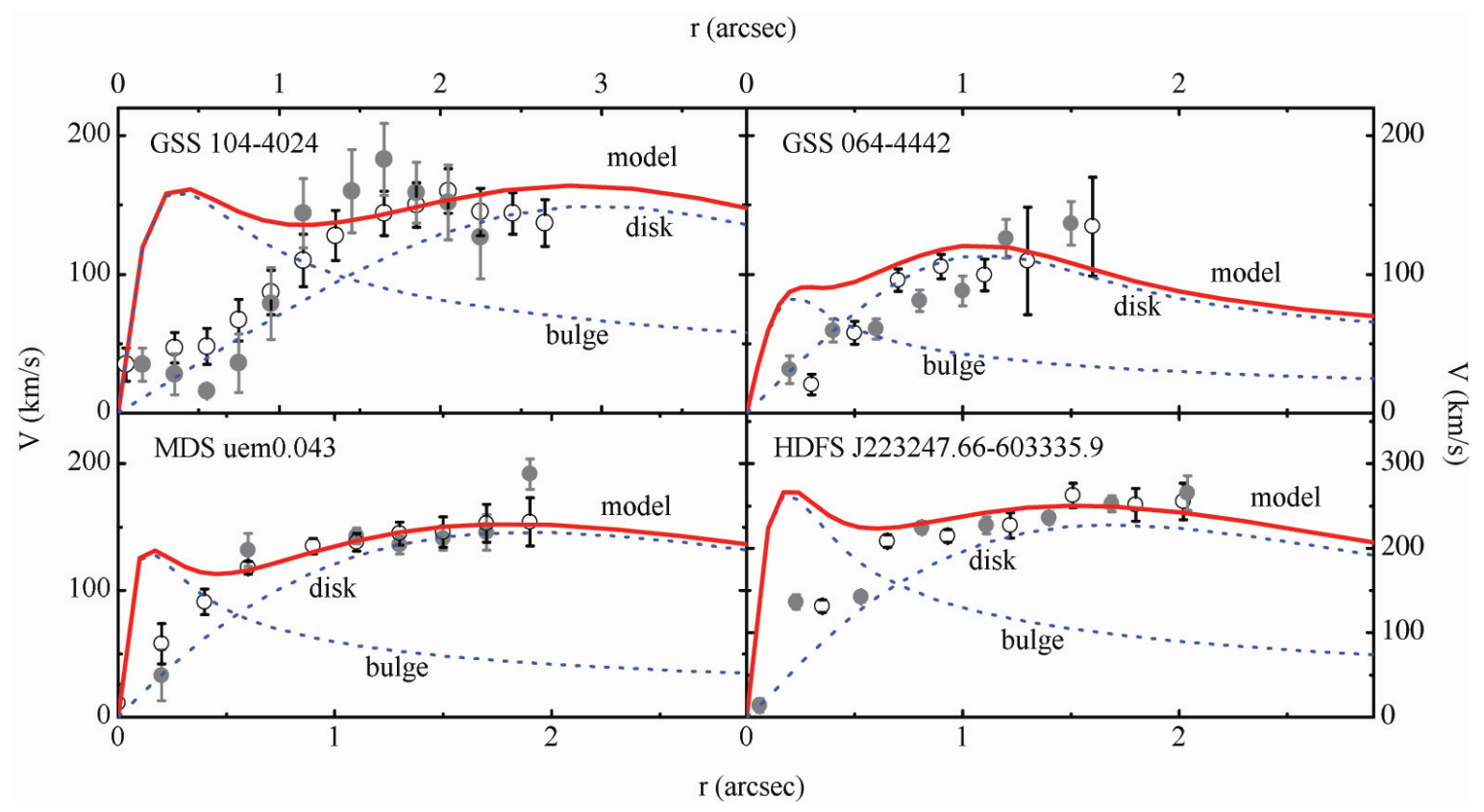

Fig. 10. Surface brightness distribution of the galaxies GSS 104-4024, GSS 064-4442, MDS uem0-043 and HDFS J223247.66-603335.9 in rest-frame $B$ color (open circles). Dashed lines - surface brightness distribution of best fit model components using spatial density distribution (2). Solid line - total surface brightness.

last observed point of rotation. The mass-to-light ratios for the disks are in this case $M / L_{B}=12 ., 1.8,1.9$ and 9.7 , respectively. Thus, within the presently available observations, the models without any dark halo fit well in most cases with the observations and it is not possible to determine the existence of a DM component in these four galaxies on the basis of their kinematics. The mean mass-to-light ratio for $z \sim 0.9$ galaxies (adding the two galaxies from Paper I) is now $\left\langle M / L_{B}\right\rangle=8.5$.

Figure 10 shows that RCs can be well fitted without DM. However, we think that there are enough independent arguments in support of the existence of DM and as a result, we think that realistic models must include a DM component.

Acknowledgements. We thank the referee, Paolo Salucci, for a number of constructive suggestions helping to improve the manuscript significantly. We thank Dr. U. Haud for making available his programs for light distribution model calculations. Surface photometry data used in the present study are from STScI Archive and we thank the members of observational proposals 5090 (PI E. Groth), 5369 (PI R. E. Griffiths) and HDFS project team members for their work. We acknowledge the financial support from the Estonian Science Foundation (grant 4702).

\section{References}

Bell, E. F., Baugh, C. M., Cole, S., Frenk, C. S., \& Lacey, C. G. 2003, MNRAS, 343, 367

Bell, E. F., Wolf, C., Meisenheimer, K., et al. 2004, ApJ, 608, 752

Berta, S., Fritz, J., Franceschini, A., Bressan, A., \& Lonsdale, C. 2004, A\&A, 418, 913

Bicker, J., Fritze-v. Alvensleben, U., Möller, C. S., \& Fricke, K. J. 2004, A\&A, 413, 37
Borriello, A., \& Salucci, P. 2001, MNRAS, 323, 285

Böhm, A., Ziegler, B., Saglia, R. P., et al. 2004, A\&A, 420, 97

Burkert, A. 1995, ApJ, 447, L25

Coleman, G. D., Wu, C.-C., \& Weedman, D. W. 1980, ApJS, 43, 393

Conselice, C. J., Bundy, K., Ellis, R. S., Brinchmann, J., \& Vogt, N. 2004, in Dark matter in galaxies, IAU Symp 220, 21-25 July 2003, ed. S. D. Ryder, D. J. Pisano, M. A. Walker, $\&$ K. C. Freeman (ASP)

Cowie, L. L., Barger, A. J., Fomalont, E. B., \& Capak, P. 2004, ApJ, 603, L69

da Costa, L. N., Nonino, M., Rengelink, R., et al. 1998 [arXiv: astro-ph/9812105]

Drory, N., Bender, R., Feulner, G., et al. 2004, ApJ, 608, 742

Einasto, J., \& Haud, U. 1989, A\&A, 223, 89

Erb, D. K., Shapley, A. E., Steidel, C. C., et al. 2003, ApJ, 591, 101

Freeman, K. C. 1970, ApJ, 160, 811

Frei, Z., \& Gunn, J. E. 1994, AJ, 108, 1476

Fukushige, T., Kawai, A., \& Makino, J. 2004, ApJ, 606, 625

Gabasch, A., Bender, R., Seitz, S., et al. 2004, A\&A, 421, 41

Gentile, G., Salucci, P., Klein, U., Vergani, D., \& Kalberla, P. 2004, MNRAS, 351, 903

Giraud, E. 1998, AJ, 116, 1125

Govenato, F., Mayer, L., Wadsley, J., et al. 2004, ApJ, 607, 688

Holtzman, J. A, Burrows, C. J., Casertano, S., et al. 1995b, PASP, 107 , 1065 (H95)

Im, M., Faber, S. M., Gebhardt, K., et al. 2001, AJ, 122, 750

Jäger, K., Ziegler, B. L., Böhm, A., et al. 2004, A\&A, 422, 907

Kelson, D. D., Illingworth, G. D., van Dokkum, P. G., \& Franx, M. 2000, ApJ, 531, 159

Khairul Alam, S. M., Bullock, J. S., \& Weinberg, D. H. 2002, ApJ, 572,34

Mazure, A., \& Capelato, H. V. 2002, A\&A, 383, 384

Milvang-Jensen, B., Aragón-Salamanca, A., Hau, G. K. T., Jørgensen, I., \& Horth, J. 2003, MNRAS, 339, L1 
Moore, B., Quinn, T., Governato, F., Stadel, J., \& Lake, G. 1999, MNRAS, 310, 1147

Navarro, J. F., \& Steinmetz, M. 2000, ApJ, 528, 607

Navarro, J. F., Frenk, C. S., \& White, S. D. M. 1996, ApJ, 462, 563

Navarro, J. F., Hayashi, E., Power, C., et al. 2004, MNRAS, 349, 1039

Pérez, I. 2004, A\&A, 427, L17

Persic, M., \& Salucci, P. 1995, ApJS, 99, 501

Persic, M., Salucci, P., \& Stel, F. 1996, MNRAS, 281, 27

Pohlen, M., Dettmar, R., \& Lütticke, R. 2000, A\&A, 357, L1

Pohlen, M., Beckman, J., Hüttemeister, S., et al. 2004, Stellar sisk truncations: where do we stand? In Penetrating bars through masks of cosmic dust, ed. D. L. Block, K. C. Freeman, I. Puerari, \& R. Gross (Kluwer) [arXiv: astro-ph/0405541]

Poli, F., Giallongo, E., Fontana, A., et al. 2003, ApJ, 593, L1

Rigopoulou, D., Franceschini, A., Aussel, H., et al. 2002, ApJ, 580, 789
Schlegel, D. J., Finkbeier, D. P., \& Davis, M. 1998, ApJ, 500, 525

Sérsic, J. L. 1968, Atlas de Galaxies Australes, Observatorio Astronomico, Cordoba, Argentina

Simard, L., Willmer, C. N. A., Vogt, N. P., et al. 2002, ApJS, 142, 1

Sofue, Y., \& Rubin, V. 2001, ARA\&A, 39, 137

Tamm, A., \& Tenjes, P. 2001, Baltic Astron., 10, 599

Tamm, A., \& Tenjes 2003, A\&A, 403, 529

Tenjes, P., Haud, U., \& Einasto, J. 1994, A\&A, 286, 753

Tenjes, P., Haud, U., \& Einasto, J. 1998, A\&A, 335, 449

Toft, S., Mainieri, V., Rosati, P., et al. 2004, A\&A, 422, 29

van Dokkum, P. G., \& Franx, M. 1996, MNRAS, 281, 985

Vogt, N. P., Forbes, D. A., Phillips, A. C., et al. 1996, ApJ, 465, L15

Wuyts, S., van Dokkum, P. G., Kelson, D. D., Franx, M., \& Illingworth, G. D. 2004, ApJ, 605, 677

Ziegler, B. L., Böhm, A., Jäger, K., Heidt, J., \& Möllenhoff, C. 2003, ApJ, 598, 87 